\title{
Dominance behaviour and division of labour in the tropical primitively eusocial wasp Ropalidia cyathiformis
}

\author{
S. Unnikrishnan ${ }^{1,2} \mathbb{( D} \cdot$ R. Gadagkar ${ }^{1} \mathbb{C}$
}

Received: 10 June 2020 / Revised: 3 October 2020 / Accepted: 2 December 2020

(c) International Union for the Study of Social Insects (IUSSI) 2021

\begin{abstract}
Primitively eusocial insects exhibit reproductive division of labour such that one or a small number of individuals monopolize reproduction while the remaining function as non-reproductive workers. They also exhibit non-reproductive division of labour such that some workers primarily perform the extra-nidal tasks of foraging, while others primarily perform the intra-nidal tasks of feeding larvae, building the nest and other nest maintenance activities. In some species, queens regulate both reproductive as well as non-reproductive division of labour by means of their dominance behavior toward the workers. Here we show that in the primitively eusocial species $R$. cyathiformis, (1) the queen shows significantly more aggression towards the potential queen (PQ) than to the rest of the workers; (2) the PQ shows significantly more aggression towards the workers than they show to each other; (3) the activities of the workers such as bringing food and feeding the larvae continue unabated in the absence of the queen; (4) the amount of dominance received by a worker does not predict her rate of foraging; (5) there is a positive correlation between workers' rates of bringing food and the rates at which they themselves feed the larvae. We suggest that while queen (along with PQ) regulates reproductive division of labour, dominance behavior is not used to regulate the non-reproductive activities of the workers such as bringing food and feeding the larvae; these are self-regulated by individual workers by themselves.
\end{abstract}

Keywords Dominance behaviour $\cdot$ Regulation of reproduction $\cdot$ Regulation of foraging $\cdot$ Primitively eusocial $\cdot$ Ropalidia cyathiformis

\section{Introduction}

Division of labour is a defining characteristic of insect societies. Division of labour can be of two kinds. First, there is reproductive division of labour such that only one or a small number of individuals (queens or kings) reproduce while the remaining colony members (workers) are prevented or

Supplementary Information The online version contains supplementary material available at https://doi.org/10.1007/s0004 0-020-00803-3.

S. Unnikrishnan

sruthiuj@gmail.com; sruthiu@ncbs.res.in

R. Gadagkar

ragh@iisc.ac.in

1 Centre for Ecological Sciences, Indian Institute of Science, Bangalore 560012, India

2 Present Address: National Centre for Biological Sciences, TIFR, GKVK, Bellary Road, Bangalore 560065, India refrain from reproduction. In primitively eusocial species such as those of some bees and wasps, dominance-subordinate interactions (hereafter, dominance behaviour, DB) are thought to play an important role in bringing about reproductive division of labour. This is inferred from the observations that in most primitively eusocial species, the queen, monopolizes egg laying in the colony, is behaviourally dominant and is usually at the top of the dominance hierarchy, and that the loss or experimental removal of the queen results in one or more of the workers beginning to lay eggs (Pardi 1948; West-Eberhard 1969; Jeanne 1972; Gadagkar and Joshi 1982; Strassmann and Meyer 1983; Hughes et al. 1987; Keeping 1990). The only exception to this rule seems to be the case of mature colonies of Ropalidia marginata where the queens are strikingly meek and docile and seldom participate in the colony's dominance subordinate interactions, although the loss of the queen nevertheless leads to egg laying by one of the workers (Gadagkar and Joshi 1983; Chandrashekara and Gadagkar 1992; Gadagkar 2001). The 
queen in this species appears to regulate worker reproduction through pheromones (Bhadra et al. 2010; Mitra et al. 2011).

Dominance behaviour by the queen is also thought to play a role in regulating non-reproductive division of labour. This has received support in the primitively eusocial wasp Polistes fuscatus where it has been reported that worker activity levels drop sharply upon queen removal and resumes upon re-introduction of the queen (Reeve and Gamboa 1983, 1987). But this might not be true for all primitively eusocial species as it has been shown in Polistes instabilis, P. dominulus (Jha et al. 2006) and P. versicolor (Souza and Prezoto 2012) as well as in Ropalidia marginata (Premnath et al. 1995, 1996; Gadagkar 2001) that the queen is not the central pacemaker of the colony as the worker activity levels do not decline in the absence of the queen. Possible alternatives to the regulation of work in the colony by the queen could be(1) decentralised mode of regulation as has been observed in highly eusocial insects such as honeybees (Seeley 1982) and stingless bees (Sommeijer 1984); (2) self-regulated mode of work regulation with the same individual performing multiples tasks and thereby gaining information on its own. Such self-regulation is perhaps what occurs in bumblebees (Cameron 1989). In Ropalidia marginata it has also been shown that work regulation occurs in a decentralised manner where workers use dominance behaviour to communicate colony hunger status to the foragers (Premnath et al. 1995, 1996; Bruyndonckx et al. 2006; Lamba et al. 2007; Gadagkar 2019).

Dominance behaviour is believed to be the mode of communication or mechanism by which work is regulated in these primitively eusocial insect groups. Whether by queen or by a decentralised manner, dominance behaviour has been linked to be the mechanism for the same. In the case of queen control, it is believed that queen uses dominance behaviour to regulate the work in the colony (Reeve and Gamboa 1983, 1987). In the case of decentralised work regulation, in $R$. marginata it has been shown that the intranidal workers use dominance behaviour to communicate the colony hunger status to the foragers (Premnath et al. 1995, 1996; Bruyndonckx et al. 2006; Lamba et al. 2007; Gadagkar 2019). Hence dominance behaviour is believed to be closely linked to the regulation of work in primitively eusocial colonies.

Ropalidia cyathiformis, the subject of the current study, exhibits features typical of primitively eusocial species such as dominance-based queen succession and weak age polyethism (Unnikrishnan and Gadagkar 2017) and contrasting with its better studied congeneric species $R$. marginata. Unlike the latter, $R$. cyathiformis has behaviourally dominant queens (Gadagkar and Joshi 1984; Deshpande et al. 2006) but as in R. marginata, removal of the queen results in one of the workers (the potential queen, PQ) becoming temporarily hyper-aggressive and taking over as the next queen in a few days (Deshpande et al. 2006). In R. cyathiformis, the potential queen not only increases her dominance behaviour in the absence of the queen, but also significantly increases her activity levels and other non-dominance interactions with her nestmates (Kardile and Gadagkar 2003). Unlike in $R$. marginata, the frequency per hour of dominance behaviour shown by workers in the presence of the queen is a significant, though not perfect, predictor of which individual will become the next queen (Unnikrishnan and Gadagkar 2017). Based on these observations, it has been speculated that in $R$. cyathiformis, the queen maintains her reproductive monopoly using dominance behaviour and also regulates the non-reproductive activities of the workers in a centralised manner, and that the potential queen takes over this role in the absence of the queen (Kardile and Gadagkar 2003).

In the present study, we examine the role of dominance behaviour in the regulation of reproductive as well as nonreproductive division of labour. We do so by analysing not only the rates of dominance behaviour shown by different individuals in the colony, but also by examining the targets of dominance behaviour.

\section{Methods}

The results reported in this paper are based on behavioural observations on 23 nests of the primitively eusocial wasp Ropalidia cyathiformis, in the campus of the Indian Institute of science, Bangalore $\left(12.97^{\circ} \mathrm{N}, 77.59^{\circ} \mathrm{E}\right)$. All adult wasps on all nests were marked with coloured spots of quick drying Testors ${ }^{\circledR}$ enamel paint, for individual identification.

On 19 nests, we made behavioural observations from 0800 to $1030 \mathrm{~h}$ and 1300 to $1530 \mathrm{~h}$ on day 1 , and from 1030 to $1300 \mathrm{~h}$ and 1530 to $1800 \mathrm{~h}$ on day 2 . Our behavioural observations consisted of equal numbers of 'scans' (in which the behavioural state of every individual was recorded at randomly chosen instants of time) and 'all occurrence sessions' (AOS) (in which every occurrence of every selected behaviour by every individual was recorded during 5 -minute intervals), randomly intermingled with each other with a 1-minute gap between two consecutive sessions. Hence at the end of 2 days we obtained $10 \mathrm{~h}$ of observations with 50 scans and 50 all occurrence sessions on the queen-right colonies. On the morning of day 3 , at $0800 \mathrm{~h}$, we removed the queen (who was identified based on egg laying during behavioural observations on days 1 and 2). We then observed the nest for $6 \mathrm{~h}$ from 0800 to $1000 \mathrm{~h}, 1100$ to $1300 \mathrm{~h}$ and 1400 to $1600 \mathrm{~h}$ to yield 30 scans and 30 AOS.

We observed four additional nests for $6 \mathrm{~h}$ each day, without removing the queen. The observation schedule alternated between $0630-0830 \mathrm{~h}, 1030-1230 \mathrm{~h}$ and $1430-1630 \mathrm{~h}$ on 1 day and $0830-1030 \mathrm{~h}, 1230-1430 \mathrm{~h}$ and $1630-1830 \mathrm{~h}$ on the other day and continued until the nest got destroyed by 
predators or other natural calamities. We observed these four nests for 15, 20, 27 and 28 days, respectively. Observations consisted of an equal number of scans and all occurrences randomly intermingled with each other, so that on each day there were 30 scans and 30 all occurrences giving a total of 300 min of observation per day.

For all 23 nests, we used the scans to estimate the proportions of time spent by each wasp in different behaviours and the AOS sessions to estimate the frequency per hour with which different wasps performed different behaviours. In all 23 nests, the queen was identified by her egg laying behaviour. In all nests, the PQ was identified based on the following two criteria-(1) she should show at least five acts of dominance behaviour and (2) she should show more dominance behaviour than any other individual in the colony (Unnikrishnan and Gadagkar 2017). In the latter set of four nests which were observed for $6 \mathrm{~h}$ every day, we did not perform quantitative behavioural observations after queen removal (which was done at the end of the study to identify the PQ). Hence our sample sizes for the comparison of the behaviour of wasps between the queen-right and queen less colonies would be 19 nests and for all other comparisons it would be 23 nests. Data from the first set of 19 nests were also used to study queen succession (Unnikrishnan and Gadagkar 2017) and data from the second set of 4 nests were also used to study age polyethism (Unnikrishnan and Gadagkar 2020). Data from all the 23 nests are being used here to study the role of dominance behaviour in division of labour. Although all the behaviours were observed during the observations in all the above 23 nests, only data on dominance behaviour, feed larva behaviour and bring food behaviour, obtained from the AOS sessions were analysed in the current study. We obtained the frequency per hour with which a behaviour was shown by dividing the total number of times the behaviour was shown by the number of hours for which that individual was observed. We divided the dominance behaviour shown by each individual by the number of wasps to which it showed dominance to obtain the per capita of DB. Information of the nests used in the analysis is given in Table S.1.

\section{Data availability}

All data from this study is made available via Dryad repository (https://doi.org/10.5061/dryad.qfttdz0fr).

\section{Statistical analysis}

We carried out all statistical analysis using $R$ version 3.4.1 ( $\mathrm{R}$ core team 2017) [with the R studio IDE ( $\mathrm{R}$ studio team 2016)]. In the first subsection, we have only used the percapita dominance behaviour shown by the individuals. We constructed linear mixed effects models using the package lme4 (Bates et al. 2015) and Emmeans (Searle et al. 2012) to make pairwise comparisons. We analysed the correlation between variables using Kendall's correlation coefficient and used Ggplot2 (Wickham 2016) to visualize the data.

\section{Results}

\section{Who shows dominance to whom?}

To understand the dominance relationships amongst the queen $(\mathrm{Q})$, potential queen $(\mathrm{PQ})$ and workers $(\mathrm{W})$, we built a linear mixed effects model with the per-capita dominance behaviour shown, as the response variable and all possible combinations of givers and receivers $(\mathrm{Q}$ to $\mathrm{PQ}, \mathrm{Q}$ to $\mathrm{W}, \mathrm{PQ}$

Table 1 Table showing the fixed effects, random effects and the estimate with confidence intervals and $P$ values for the linear mixed effects model studying the per-capita dominance behaviour shown by Q, PQ and W

\begin{tabular}{lccc}
\hline Fixed effects & Estimate & CI & $P$ value \\
\hline (Intercept) PQ-Q & 0.17391 & -2.33 to 2.67 & 0.8910 \\
giv.recPQ-W & 4.21119 & 0.8 to 7.62 & $\mathbf{0 . 0 1 6 1}$ \\
giv.recQ-PQ & 8.47826 & 5.07 to 11.89 & $\mathbf{0 . 0 0 0 1}$ \\
giv.recQ-W & 0.53888 & -2.87 to 3.95 & 0.7558 \\
giv.recW-PQ & -0.13745 & -3.55 to 3.28 & 0.9368 \\
giv.recW-Q & -0.14070 & -3.55 to 3.27 & 0.9353 \\
giv.recW-W & -0.09162 & -3.5 to 3.32 & 0.9578 \\
\hline Random effects & Variance & & \\
\hline Nest & 2.558 & & \\
Residual & 34.383 & & \\
\hline
\end{tabular}

Dominance behaviour shown by PQ to W and Q to PQ are significantly different from the intercept. Fixed effect, the identity of the giver and receiver of dominance is a categorical variable and the per-capita DB is the response variable which is a continuous variable

Bold specifies $p$ values less than 0.05 
to $\mathrm{Q}, \mathrm{PQ}$ to $\mathrm{W}, \mathrm{W}$ to $\mathrm{Q}, \mathrm{W}$ to $\mathrm{PQ}$ and $\mathrm{W}$ to $\mathrm{W}$ ) of dominance behaviour as the predictor variables and the nest identity as a random effect (Table 1). We found that the per-capita dominance behaviour shown by $\mathrm{Q}$ to $\mathrm{PQ}$ and that shown by $\mathrm{PQ}$ to $\mathrm{W}$ were significantly higher than the rest of the combinations (Fig. 1, Tables 1,2).

\section{Does the absence of the queen matter?}

We built two separate linear mixed effects models with the rate of feeding larva behaviour and the rate of bring food as response variables, and the presence $(\mathrm{QR})$ or absence of the queen $(\mathrm{QL})$ as a fixed effect and the nest id as a random effect. We found no significant difference in the rate of feed larva and in the rate of bring food behaviours between QR and QL conditions (Fig. 2, Tables 3 and 4). Thus, two of the most fundamental activities of the nest did not show any decline in their rates in the absence of the queen.
Table 2 Table showing the results of the paired-wise analysis of the linear mixed effects model to understand which combination of giverreceiver are different from each other

\begin{tabular}{lrlr}
\hline Contrast & Estimate & CI & $P$ value \\
\hline Q-PQ vs Q-W & 7.79393 & 4.445 to 11.43 & $<\mathbf{0 . 0 0 0 1}$ \\
Q-PQ vs PQ-W & 4.26707 & 0.773 to 7.76 & $\mathbf{0 . 0 1 7 1}$ \\
Q-PQ vs W-W & 8.56988 & 5.075 to 12.06 & $<\mathbf{0 . 0 0 0 1}$ \\
PQ-W vs PQ-Q & 4.21119 & 0.717 to 7.71 & $\mathbf{0 . 0 1 8 5}$ \\
PQ-W vs Q-W & 4.30281 & 0.178 to 7.17 & $\mathbf{0 . 0 3 9 6}$ \\
PQ-W vs W-W & 4.30281 & 0.808 to 7.8 & $\mathbf{0 . 0 1 6 2}$ \\
W-Q vs W-W & -0.04908 & -3.544 to 3.45 & 0.9779 \\
W-PQ vs W-W & -0.00326 & -3.498 to 3.49 & 0.9985 \\
\hline
\end{tabular}

Dominance behaviour shown by $\mathrm{Q}$ to $\mathrm{PQ}$ is significantly different from $\mathrm{Q}$ to $\mathrm{W}, \mathrm{PQ}$ to $\mathrm{W}$ and $\mathrm{W}$ to $\mathrm{W}$. The dominance behaviour shown by $\mathrm{PQ}$ to $\mathrm{W}$ is significantly different from $\mathrm{PQ}$ to $\mathrm{Q}, \mathrm{Q}$ to $\mathrm{W}$ and $\mathrm{W}$ to $\mathrm{W}$

Bold specifies $p$ values less than 0.05
Fig. 1 Per-capita dominance behaviour shown by the potential queen $(\mathrm{PQ})$, queen $(\mathrm{Q})$ and workers $(\mathrm{W})$ to each other. The per-capita dominance behaviour shown by Q to PQ (LMM - Estimate $=8.478$, CI 5.07-11.89, $P$ value $<0.0001$ ) and $\mathrm{PQ}$ to $\mathrm{W}$ $($ LMM - Estimate $=4.2112$, CI 0.8-7.62, $P$ value $=0.016$ ) was significantly higher than the intercept. The per-capita DB by Q to PQ was also higher than that shown by Q to $\mathrm{W}$ (Estimate $=7.94$, CI 4.4-11.4, $P$ value $<0.0001)$, and $\mathrm{W}$ to $\mathrm{W}$ (Estimate $=8.57$, CI 5.1-12.1, $P$ value $<0.0001)$. The percapita DB shown by PQ to W was higher than that shown by $\mathrm{PQ}$ to $\mathrm{Q}$ (Estimate $=4.21, \mathrm{CI}$ $0.72-7.7, P$ value $=0.018) \mathrm{W}$ to $\mathrm{W}$ (Estimate $=4.3$, CI 0.8-7.8, $P$ value $=0.016)$ and $\mathrm{Q}$ to $\mathrm{W}$ (Estimate $=3.67$, CI 0.18-7.2, $P$ value $=0.039) . N=23$ colonies

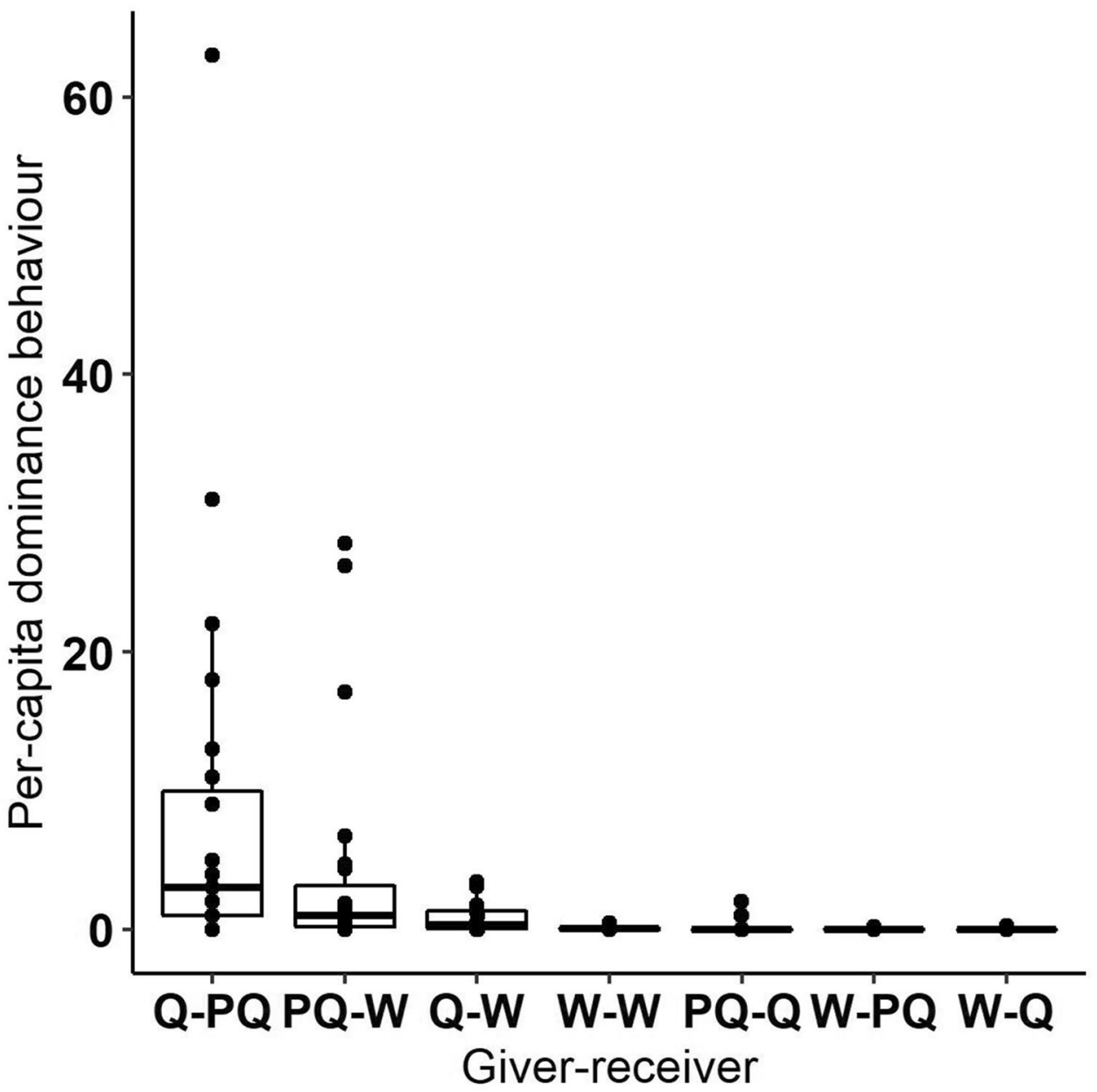


Fig. 2 Frequency per hour of feed larva (FL) (left panel) and bring food (BF) (right panel) behaviours in queen-right $(\mathrm{QR})$ and queen-less (QL) sessions. There was no difference in the frequency of FL between QR and QL sessions (LMM Estimate $=0.05, \mathrm{CI}-0.07$ to $0.17, P$ value $=0.39)$ and also in frequency of BF between QR and QL sessions (LMM Estimate $=-0.002, \mathrm{CI}-0.06$ to $0.05, P$ value $=0.94) . N=19$ colonies

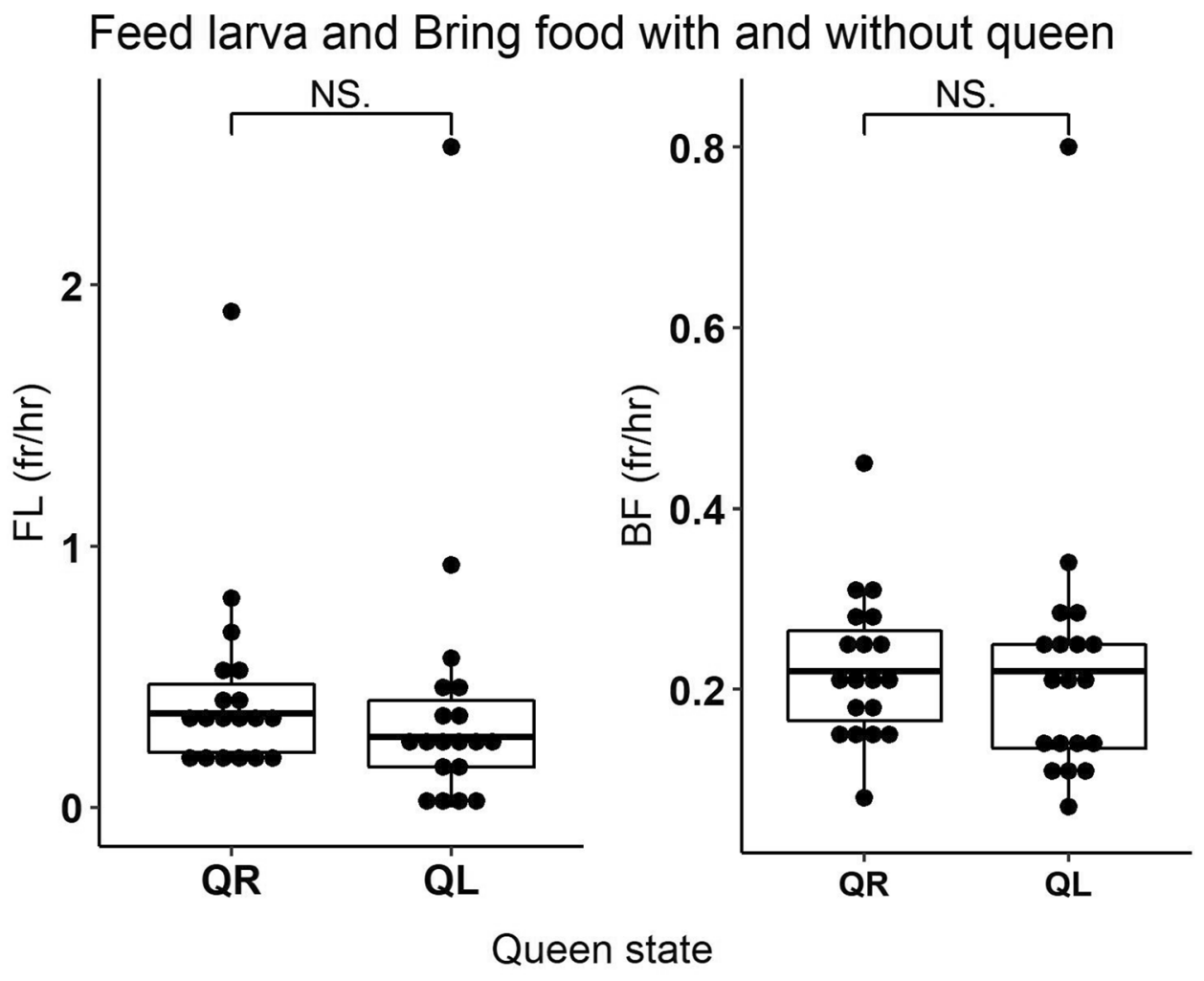

Table 3 Table showing the fixed effects, random effects and the estimate with confidence intervals and $P$ values for the linear mixed effects model studying the effect of queen right $(\mathrm{QR})$ and queen less $(\mathrm{QL})$ condition on feed larva behaviour

\begin{tabular}{llll}
\hline Fixed effects & Estimate & CI & $P$ value \\
\hline (Intercept) QL & 0.3968 & 0.18 to 0.62 & -0.07 to 0.17 \\
QR & 0.0511 & & 0.001 \\
\hline Random effects & Variance & \\
\hline Nest & 0.19 & \\
Residual & 0.032 & & \\
\hline
\end{tabular}

There is no significant difference in feed larva behaviour between queen right and queen less conditions. The fixed effect which is the presence or absence of the queen is a categorical variable and the response variable is feed larva behaviour is a continuous variable

Bold specifies $p$ values less than 0.05

Table 4 Table showing the fixed effects, random effects and the estimate with confidence intervals and $P$ values for the linear mixed effects model studying the effect of queen right (QR) and queen less (QL) condition on bring food behaviour

\begin{tabular}{lccc}
\hline Fixed effects & Estimate & CI & $P$ value \\
\hline (Intercept) QL & 0.2268 & 0.17 to 0.28 & $<.0001$ \\
QR & -0.002 & -0.06 to 0.05 & 0.937 \\
\hline Random effects & Variance & \\
\hline Nest & 0.008 & & \\
Residual & 0.007 & & \\
\hline
\end{tabular}

There is no significant difference in bring food behaviour between queen right and queen less conditions. The fixed effect which is the presence or absence of queen is a categorical variable. Bring food behaviour is the response variable and is a continuous variable

Bold specifies $p$ values less than 0.05 
Fig. 3 Shows the regression between dominance behaviour received and bring food behaviour (BF). There was no significant effect of dominance received on the bring food behaviour of wasps (LMM Estimate $=-0.0004, \mathrm{CI}-0.003$ to $0.002, P$ value $=0.752$ ). Data points represent $\mathrm{fr} / \mathrm{hr}$ of $\mathrm{BF}$ for each forager for that particular amount of dominance received for 23 colonies

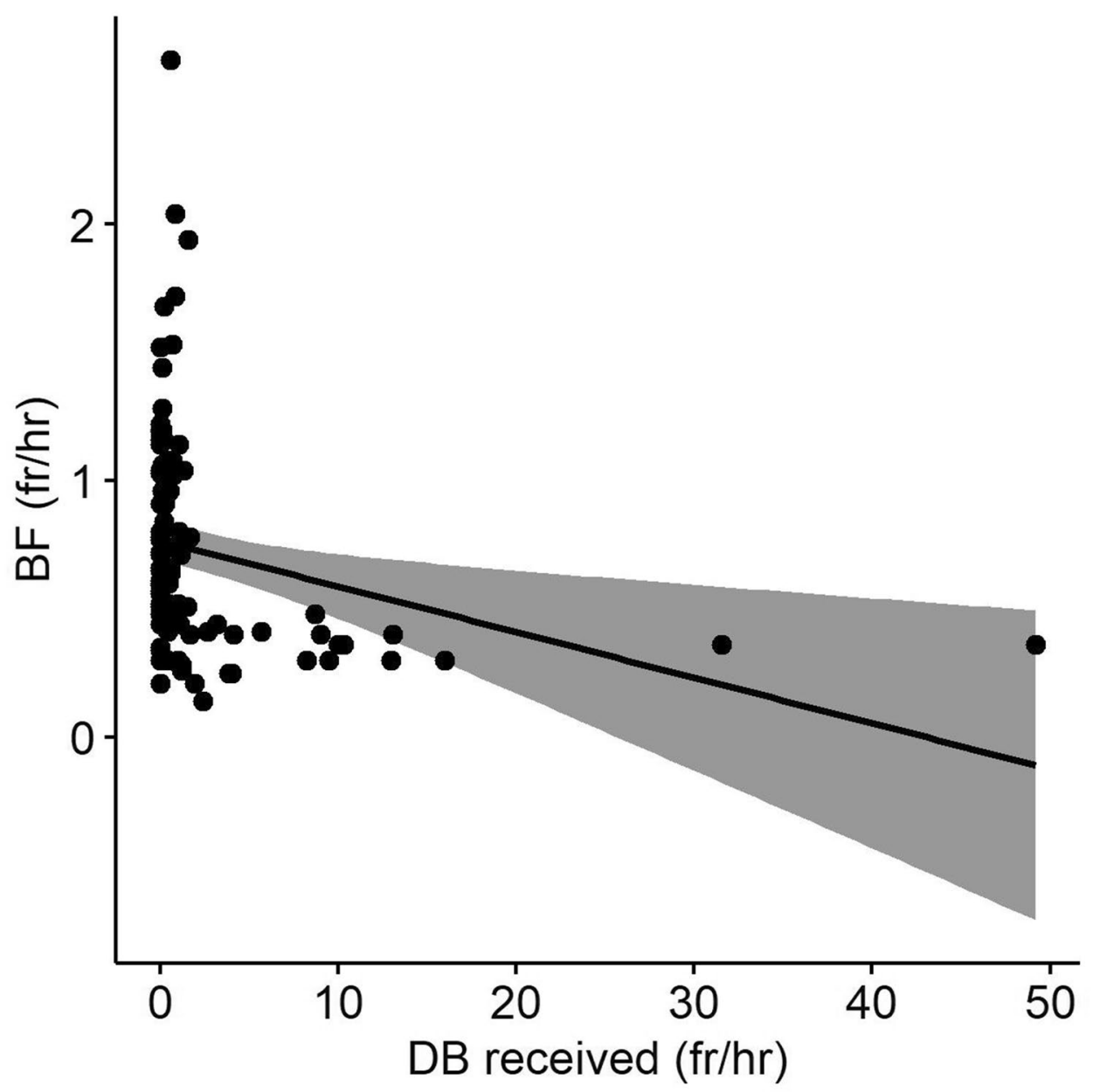

Table 5 Table showing the fixed effects, random effects and the estimate with confidence intervals and $P$ values for the linear mixed effects model studying the effect of dominance behaviour received on bring food behaviour

\begin{tabular}{lccc}
\hline Fixed effects & Estimate & CI & $P$ value \\
\hline (Intercept) & 0.8026 & 0.58 to 1.02 & -0.003 to 0.002 \\
DB received & -0.0004 & & 0.752 \\
\hline Random effects & Variance & \\
\hline Nest & 0.27 & \\
Residual & 0.0037 &
\end{tabular}

There is no significant effect of dominance received on the individual bring food behaviour. Bring Food behaviour is the response variable and $\mathrm{DB}$ received is the fixed effect. Both are continuous variables

Bold specifies $p$ values less than 0.05

\section{Does dominance received predict the rate of bringing food?}

To see if the rate of dominance behaviour received by individual wasps had any effect on their rate of bringing food, we built a generalised linear mixed effects model with rate of bring food behaviour of each individual as the response variable and the rate of dominance behaviour received by that individual as the predictor variable and the nest identity as a random effect. We found no significant effect of dominance received on the rate of bring food behaviour (Fig. 3, Table 5). Since the dominance received by many individuals was zero, we also compared the rates of bringing food by wasps that received no DB versus those that 
Fig. 4 The amount of bring food behaviour when no dominance $(\mathrm{DB})$ is received $(\mathrm{N})$ and when some amount of $\mathrm{DB}$ is received $(\mathrm{Y})$. There is no significant difference in bring food amongst individuals that did not receive any DB $(36$ wasps) and those that received some DB (77 wasps) (LMM, Estimate $=0.015, \mathrm{CI}-0.013$ to $0.044, P$ value $=0.28) . N=23$ colonies

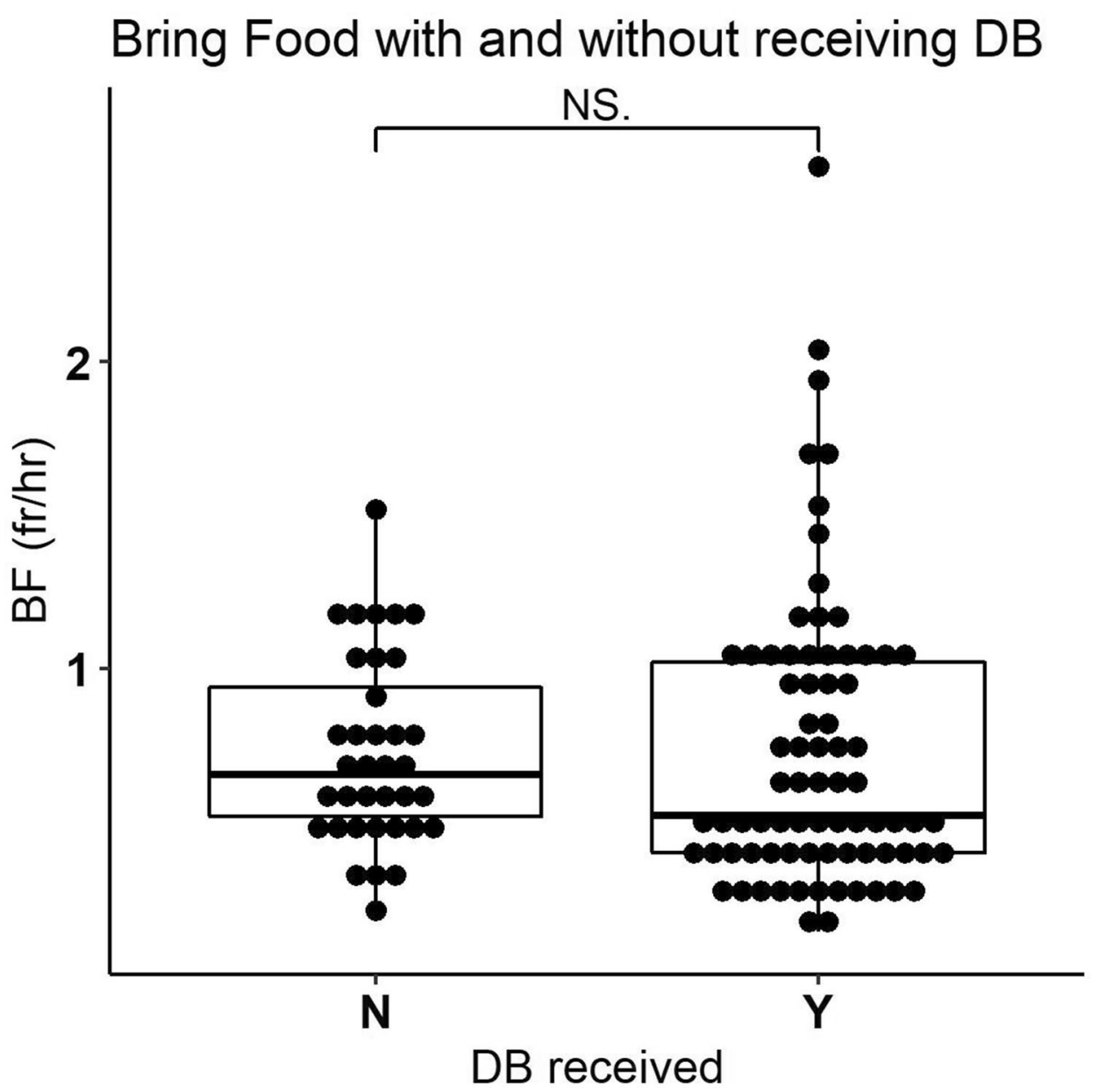

Table 6 Table showing the fixed effects, random effects and the estimate with confidence intervals and $P$ values for the linear mixed effects model studying the bring food behaviour when DB is not received $(\mathrm{N})$ as opposed to when some dominance is received $(\mathrm{Y})$

\begin{tabular}{lllc}
\hline Fixed effects & Estimate & CI & $P$ value \\
\hline (Intercept) N & 0.7912 & 0.57 to 1.01 & $<\mathbf{0 . 0 0 0 1}$ \\
DB received Y & 0.0156 & -0.013 to 0.04 & 0.282 \\
\hline Random effects & Variance & \\
\hline Nest & 0.27 & \\
Residual & 0.004 & & \\
\hline
\end{tabular}

There is no significant difference in bring food behaviour between those that receive and do not receive any DB. The fixed effect variable, whether DB received or not is a categorical variable and bring food behaviour is the response variable, which is a continuous variable

Bold specifies $p$ values less than 0.05

received some DB, treating DB received or not as a binary variable. Of the 113 wasps studied, 36 wasps received no dominance behaviour at all and yet brought food at rates comparable to the remaining 77 wasps that received varying amounts of dominance behaviour during the observations Fig. 4, Table 6).

\section{Do the foragers feed larvae?}

To check whether forgers themselves feed the larvae rather than depending entirely on intranidal workers to do so, we analysed the correlation between the rates of bring food behaviour and feed larva behaviour of different wasps. We found a weak but significantly positive correlation between 


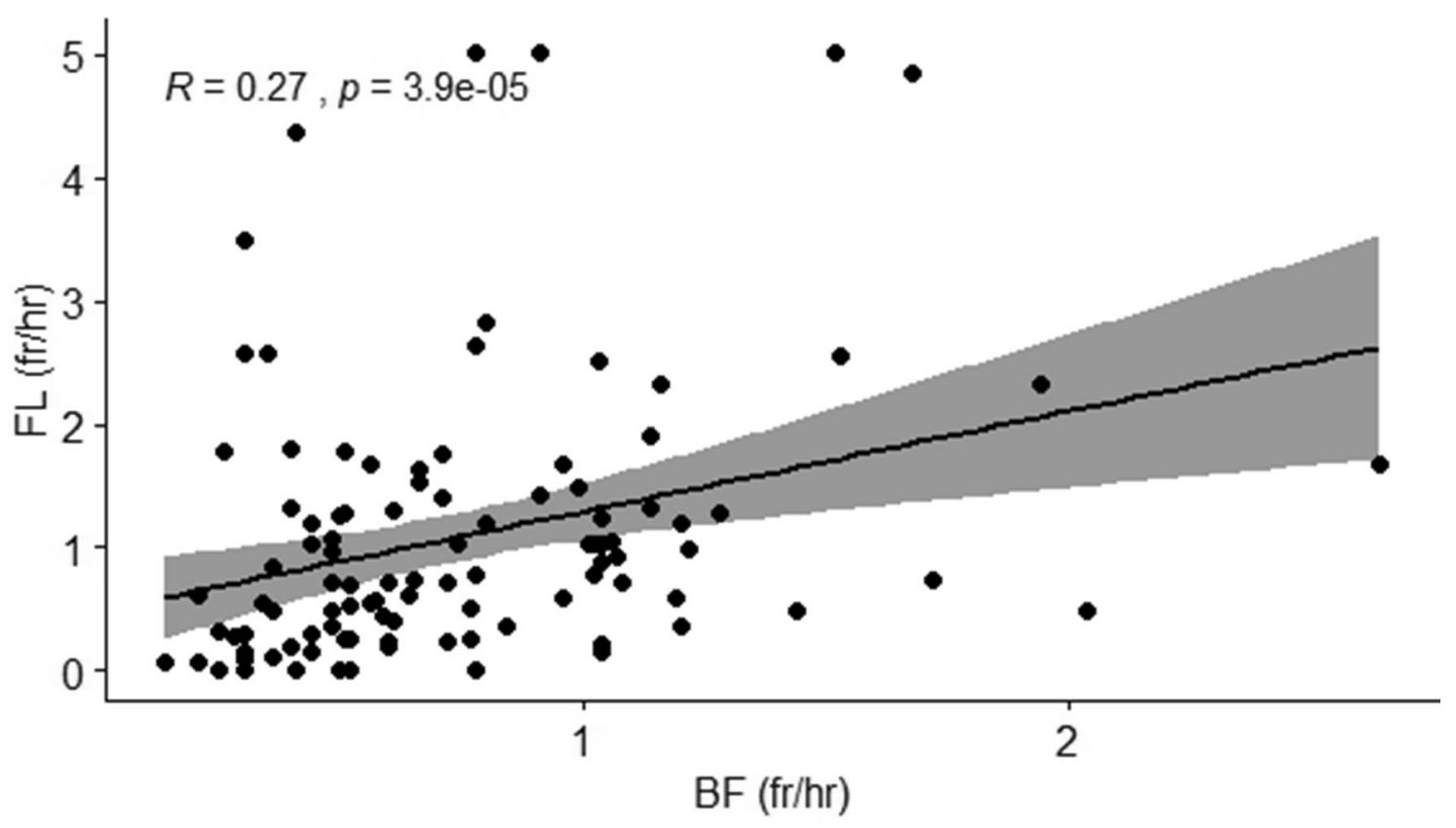

Fig. 5 Visualisation of correlation between Bring Food (BF) behaviour and Feed Larva (FL) behaviour. Kendall's correlation coefficient, $\tau=0.27, P<0.0001$. Data points represent $\mathrm{fr} / \mathrm{hr}$ of FL for each forager for that particular freq $/ \mathrm{hr}$ of $\mathrm{BF}$ for 23 colonies

the rates at which individuals brought food and the rates at which they fed the larvae (Fig. 5).

\section{Discussion}

In several species of primitively eusocial wasps the queen is usually considered as the alpha individual and the future queen (PQ, in our terminology) as the beta individual. In some studies, this means that the queen shows the highest rates of dominance behaviour in the colony and the PQ shows the next highest rate. In other studies, it means that the queen occupies the top rank in the dominance hierarchy of the colony so that she is dominant over everyone else and no one is dominant over her. Similarly the PQ occupies the next highest rank, so that she dominates everyone else except the queen (Pardi 1948; West-Eberhard 1969; Jeanne 1972; Litte 1979; Strassmann and Meyer 1983; Hughes et al. 1987; Hughes and Strassmann 1988; Keeping 1992, 1997; Giannotti and Machado 1997).

The results presented in this paper may permit us to draw some inferences regarding the possible role of dominance behaviour in regulating both reproductive and non-reproductive division of labour in the primitively eusocial wasp $R$. cyathiformis. We find that the per capita rates of dominance behaviour shown by the queen to the PQ and that shown by the PQ to the workers, are both higher than the corresponding per-capita rates among the workers themselves. This suggests that the queen's dominance behaviour towards the
PQ and the PQ's dominance behaviour towards the workers may together help the queen to maintain her reproductive monopoly as the sole egg layer in the colony. Since the PQ is next in line to become the queen of the colony and she is therefore the greatest threat to the queen, it is not surprising that the queen directs most of her dominance to the PQ. Since the rest of the workers are a potential threat to the PQ's prospects of becoming the next queen, it is equally unsurprising that the PQ directs most of her dominance to the rest of the workers. When the PQ becomes the next queen and one of the workers becomes the next PQ and they follow similar queen- and PQ-specific patterns of dominance behaviour, reproductive division of labour and the queen's monopoly on egg laying can continue smoothly through a series of queen successions, as if often witnessed in colonies of this tropical species.

Our results also permit some inferences regarding the role of dominance behaviour in the regulation of non-reproductive division of labour. We find that the important activities of the workers such as bringing food and feeding the larvae continue unabated after the removal of the queen, suggesting that the queen is not regulating these activities of the workers through her dominance behaviour, as a central pacemaker as has been suggested for some species (Reeve and Gamboa 1983, 1987) but not in others (Jha et al. 2006; Souza and Prezoto 2012). This difference could also be attributed to the fact that the methodology of the studies was different. In the studies by Reeve and Gamboa (1983, 1987), queen although cooled and inactive, was still present 
on the nest while the queen was completely removed in the other studies as is the case in the current study as well. This might perhaps have led to differences in the results between these different studies.

It is true that we have observed the unaltered behaviour of the workers only for 1 day after queen removal. However, we think it is unlikely that they will alter their behaviour on subsequent days because the maximum disruption to the colony due to the dominance behaviour of the PQ is on day 1 after the queen removal. The PQ appears to begin to behave like a typical queen soon after this; indeed, she has been observed to lay her first egg as early as 1 day after queen removal (Unnikrishnan and Gadagkar 2017).

Kardile and Gadagkar (2003) had previously speculated that the queen may act as the central pacemaker in R. cyathiformis and the PQ may take over that role after the death of the queen. However, we now find that the levels of dominance received by the workers do not predict their foraging levels. On the other hand, we find a weak, but significant positive correlation between the rates at which individual wasps bring food and their own rates of feeding the larvae. We therefore speculate that foragers in this species may regulate their own foraging levels by obtaining direct information about larval hunger levels through their significant contribution to larval feeding. This is in contrast to $R$. marginata where workers appear to regulate each other's foraging through dominance behaviour by a decentralized self-organized manner (Premnath et al. 1995; Bruyndonckx et al. 2006; Lamba et al. 2007; Gadagkar 2019). The lack of decentralised work regulation and presence of self-regulated foraging in $R$. cyathiformis is consistent with the lack of clear division of labour between intranidal and extranidal tasks in this species (Unnikrishnan and Gadagkar 2020).

In addition to bringing food to the colony the workers also bring liquid and pulp to the colony. In this study, we have focused on bring food behaviour and feed larva behaviour, taking one extranidal and one intranidal tasks to understand how work regulation might happen in this species. It would be interesting to see what kind of pattern or correlation might be present between bring liquid, bring pulp and construction behaviour, in future studies.

In summary, dominance behaviour appears to have a role in regulating reproductive division of labour but not nonreproductive division of labour in $R$. cyathiformis, quite in contrast to the congeneric $R$. marginata where dominance behaviour appears to regulate non-reproductive division of labour but not reproductive division of labour. With its pheromonal control of reproduction and decentralized, self-organized regulation of foraging, $R$. marginata may be thought of as being relatively more similar to highly eusocial species while with its centralized control of reproductive division of labour and self-regulation of forging, $R$. cyathiformis may be thought of as being relatively closer to a typical primitively eusocial species. We have previously shown that queen succession is based more on dominance behaviour in R. cyathiformis (Unnikrishnan and Gadagkar 2017) while it is more based on age in $R$. marginata (Bang and Gadagkar 2012). Similarly, age polyethism is relatively weak and rigid in $R$. cyathiformis (Unnikrishnan and Gadagkar 2020) while it is better developed and more flexible in $R$. marginata (Naug and Gadagkar 1998). Such comparisons between $R$. cyathiformis and $R$. marginata reveal that $R$. cyathiformis promises to be an ideal model system to understand the mechanisms of social organization and division of labour in typical primitively eusocial species.

Acknowledgements This work was supported by grants (to RG) from the Department of Science and Technology (including DST-FIST program), Department of Biotechnology (including DBT-IISc Partnership Program), Council of Scientific and Industrial Research, and Ministry of Environment, Forests and Climate Change, Government of India. SU was supported by a Research Fellowship from the University Grants Commission. SU and RG designed the study, SU conducted the study and $\mathrm{SU}$ and RG co-wrote the paper

Author contributions SU and RG designed the study, SU conducted the study and SU and RG co-wrote the paper.

Funding This work was supported by grants (to RG) from the Department of Science and Technology (including DST-FIST program), Department of Biotechnology (including DBT-IISc Partnership Program), Council of Scientific and Industrial Research, and Ministry of Environment, Forests and Climate Change, Government of India. SU was supported by a Research Fellowship from the University Grants Commission.

Availability of data and material All data from this study can be accessed from Dryad repository https://doi.org/10.5061/dryad.qfttdz0fr

\section{Compliance with ethical standards}

Conflict of interest The author declares that there is no competing interest.

Consent for publication All the authors for this paper have given their consent for publication.

\section{References}

Bang A, Gadagkar R (2012) Reproductive queue without overt conflict in the primitively eusocial wasp Ropalidia marginata. Proc Natl Acad Sci USA 109:14494-14499. https://doi.org/10.1073/ pnas. 1212698109

Bates D, Mächler M, Bolker B, Walker S (2015) Fitting linear mixedeffects models using. J Stat Software 67(1):1-48. https://doi. org/10.18637/jss.v067.i01

Bhadra A, Mitra A, Deshpande SA et al (2010) Regulation of reproduction in the primitively eusocial wasp Ropalidia marginata: on the 
trail of the queen pheromone. J Chem Ecol 36:424-431. https:// doi.org/10.1007/s10886-010-9770-x

Bruyndonckx N, Kardile SP, Gadagkar R (2006) Dominance behaviour and regulation of foraging in the primitively eusocial wasp Ropalidia marginata (Lep.) (Hymenoptera: Vespidae). Behav Process 72:100-103. https://doi.org/10.1016/j.beproc.2005.11.013

Cameron SA (1989) Temporal patterns of division of labor among workers in the primitively eusocial bumble bee, Bombus griseocollis (Hymenoptera: Apidae). Ethology 80:137-151. https://doi. org/10.1111/j.1439-0310.1989.tb00735.x

Chandrashekara K, Gadagkar R (1992) Queen succession in the primitively eusocial tropical wasp Ropalidia marginata (Lep.) (Hymenoptera: Vespidae). J Insect Behav 5:193-209. https://doi. org/10.1007/BF01049289

Deshpande SA, Sumana A, Surbeck M, Gadagkar R (2006) Wasp who would be queen: a comparative study of two primitively eusocial species. Curr Sci 91:332-336

Gadagkar R (2001) The Social Biology of Ropalidia marginata: toward understanding the evolution of eusociality. Harvard University Press, Cambridge

Gadagkar R (2019) How to design experiments in animal behaviour? Why do wasps fight? Reson J Sci Educ Indian Acad Sci 25:1413-1426

Gadagkar R, Joshi NV (1982) Behaviour of the Indian social wasp Ropalidia cyathiformis on a nest of separate combs (Hymenoptera: Vespidae). J Zool 198:27-37. https://doi. org/10.1111/j.1469-7998.1982.tb02058.x

Gadagkar R, Joshi NV (1983) Quantitative ethology of social wasps: time-activity budgets and caste differentiation in Ropalidia marginata (Lep.) (Hymenoptera: Vespidae). Anim Behav 31:26-31. https://doi.org/10.1016/S0003-3472(83)80170-5

Gadagkar R, Joshi NV (1984) Social organisation in the Indian wasp Ropalidia cyathiformis (Fab.) (Hymenoptera: Vespidae). Z Für Tierpsychol 64:15-32. https://doi.org/10.1111/j.1439-0310.1984. tb00350.x

Giannotti E, Machado VLL (1997) Queen replacement in post-emergent colonies of the social wasp, Polistes lanio (Hymenoptera, Vespidae). Rev Bras Entomol 41:9-11

Hughes CR, Strassmann JE (1988) Age is more important than size in determining dominance among workers in the primitively eusocial wasp, Polistes instabilis. Behaviour 107:1-14

Hughes CR, Beck MO, Strassmann JE (1987) Queen succession in the social wasp, Polistes annularis. Ethology 76:124-132. https://doi. org/10.1111/j.1439-0310.1987.tb00678.x

Jeanne RL (1972) Social biology of the neotropical wasp Mischocyttarus drewseni. Bull Mus Comp Zool 144:63-150

Jha S, Casey-Ford RG, Pedersen JS et al (2006) The queen is not a pacemaker in the small-colony wasps Polistes instabilis and $P$. dominulus. Anim Behav 71:1197-1203. https://doi.org/10.1016/j. anbehav.2005.11.005

Kardile S, Gadagkar R (2003) regulation of worker activity in the primitively eusocial wasp Ropalidia cyathiformis. Behaviour 140:1219-1234. https://doi.org/10.1163/156853903771980567

Keeping MG (1990) Colony foundation and nestmate recognition in the social wasp, Belonogaster petiolata. Ethology 85:1-12. https ://doi.org/10.1111/j.1439-0310.1990.tb00380.x

Keeping MG (1992) Social organization and division of labour in colonies of the polistine wasp, Belonogaster petiolata. Behav Ecol Sociobiol 31:211-224. https://doi.org/10.1007/BF00168649

Keeping MG (1997) Social behavior and brood decline in reproductivephase colonies of Belonogaster petiolata (Degeer) (Hymenoptera:
Vespidae). J Insect Behav 10:265-278. https://doi.org/10.1007/ BF02765559

Lamba S, Kazi YC, Deshpande S et al (2007) A possible novel function of dominance behaviour in queen-less colonies of the primitively eusocial wasp Ropalidia marginata. Behav Process 74:351-356. https://doi.org/10.1016/j.beproc.2006.12.003

Litte M (1979) Mischocyttarus flavitarsis in Arizona: social and nesting biology of a polistine wasp. Z Für Tierpsychol 50:282-312. https ://doi.org/10.1111/j.1439-0310.1979.tb01033.x

Mitra A, Saha P, Chaoulideer ME et al (2011) Chemical communication in Ropalidia marginata: Dufour's gland contains queen signal that is perceived across colonies and does not contain colony signal. J Insect Physiol 57:280-284. https://doi.org/10.1016/j.jinsp hys.2010.11.014

Naug D, Gadagkar R (1998) The role of age in temporal polyethism in a primitively eusocial wasp. Behav Ecol Sociobiol 42:37-47

Pardi L (1948) Dominance order in Polistes wasps. Physiol Zool 21:1-13

Premnath S, Sinha A, Gadagkar R (1995) Regulation of worker activity in a primitively eusocial wasp, Ropalidia marginata. Behav Ecol 6:117-123. https://doi.org/10.1093/beheco/6.2.117

Premnath S, Sinha A, Gadagkar R (1996) Dominance relationship in the establishment of reproductive division of labour in a primitively eusocial wasp (Ropalidia marginata). Behav Ecol Sociobiol 39:125-132. https://doi.org/10.1007/s002650050274

Reeve HK, Gamboa GJ (1983) Colony activity integration in primitively eusocial wasps: the role of the queen (Polistes fuscatus, Hymenoptera: Vespidae). Behav Ecol Sociobiol 13:63-74. https ://doi.org/10.1007/BF00295077

Reeve HK, Gamboa GJ (1987) Queen regulation of worker foraging in paper wasps: a social feedback control system (Polistes fuscatus, Hymenoptera: Vespidae). Behaviour 102:147-167

Searle SR, Speed FM, Milliken GA (2012) Population marginal means in the linear model: an alternative to least squares means. Am Statistician 34(4):216-221. https://doi.org/10.1080/00031 305.1980.10483031

Seeley TD (1982) Adaptive significance of the age polyethism schedule in honeybee colonies. Behav Ecol Sociobiol 11:287-293. https:// doi.org/10.1007/BF00299306

Sommeijer MJ (1984) Distribution of labour among workers of Melipona favosa F.: age-polyethism and worker oviposition. Insect Soc 31:171-184. https://doi.org/10.1007/BF02232713

Souza ARD, Prezoto F (2012) Regulation of worker activity in the social wasp Polistes versicolor. Insect Soc 59:193-199. https:// doi.org/10.1007/s00040-011-0204-8

Strassmann JE, Meyer DC (1983) Gerontocracy in the social wasp, Polistes exclamans. Anim Behav 31:431-438. https://doi. org/10.1016/S0003-3472(83)80063-3

Unnikrishnan S, Gadagkar R (2017) Dominance based reproductive queue in the primitively eusocial wasp, Ropalidia cyathiformis. Insect Soc 64:495-503. https://doi.org/10.1007/s0004 0-017-0568-5

Unnikrishnan S, Gadagkar R (2020) The effect of age on non-reproductive division of labour in the tropical primitively eusocial wasp, Ropalidia cyathiformis. Int J Dev Biol 64:277-283. https://doi. org/10.1387/ijdb.190213su

West-Eberhard MJ (1969) The social biology of polistine wasps. Museum of Zoology, University of Michigan, Ann Arbor

Wickham H (2016) ggplot2: elegant graphics for data analysis. Springer-Verlag New York. ISBN 978-3-319-24277-4. https:// ggplot2.tidyverse.org 